\title{
External polarimetric calibration of the Danish polarimetric C-band SAR
}

\author{
Skriver, Henning; Dall, Jørgen; Madsen, Søren Nørvang
}

Published in:

Proceedings of the International Geoscience and Remote Sensing Symposium

Link to article, DOI:

10.1109/IGARSS.1994.399357

Publication date:

1994

Document Version

Publisher's PDF, also known as Version of record

Link back to DTU Orbit

Citation $(A P A)$ :

Skriver, H., Dall, J., \& Madsen, S. N. (1994). External polarimetric calibration of the Danish polarimetric C-band SAR. In Proceedings of the International Geoscience and Remote Sensing Symposium (Vol. Volume 2, pp. 1105-1107). IEEE. https://doi.org/10.1109/IGARSS.1994.399357

\section{General rights}

Copyright and moral rights for the publications made accessible in the public portal are retained by the authors and/or other copyright owners and it is a condition of accessing publications that users recognise and abide by the legal requirements associated with these rights.

- Users may download and print one copy of any publication from the public portal for the purpose of private study or research.

- You may not further distribute the material or use it for any profit-making activity or commercial gain

- You may freely distribute the URL identifying the publication in the public portal 


\title{
External Polarimetric Calibration of the Danish Polarimetric C-band SAR
}

\author{
Henning Skriver ${ }^{1}$, Jørgen Dall ${ }^{2}$, and Søren Nørvang Madsen ${ }^{3}$ \\ Electromagnetics Institute, Technical University of Denmark \\ DK-2800 Lyngby Denmark \\ T: $+4542881444 \quad$ F: +4545931634 \\ EMail: 1hs@emi.dtu.dk2jd@emi.dtu.dk3snm@emi.dtu.dk
}

\begin{abstract}
Results of applying two cross-talk removal algorithms (Klein's and Quegan's methods) based on natural distributed targets to the Danish polarimetric airborne SAR (EMISAR) are presented. Also, a new approach of obtaining robust estimates by the algorithms is proposed. The approach is based on a masking procedure using the cross-correlation between co- and cross-polarized returns. The cross-talk estimated for the EMISAR data is low (below -30 dB), virtually independent of range, and stable between different acquisitions - within a mission and between missions. The cross-talk is reduced by $8 \mathrm{~dB}$ after correction to less than $-38 \mathrm{~dB}$.
\end{abstract}

\section{INTRODUCTION}

A research and development project initiated in 1986 at the Electromagnetics Institute (EMI) of the Technical University of Denmark has resulted in a C-band VV polarized airborne SAR first flown in 1989 (Madsen et al., 1991) and later a fully polarimetric system, which has successfully acquired data since 1993 (Christensen et al., 1994). The SAR is presently being extended to dual-frequency (L- and C-band) capability expected to be operated from the beginning of 1995 . The polarimetric extensions of the system has been cosponsored by the EU's Joint Research Centre (JRC). The Danish airborne polarimetric SAR (EMISAR) is used for scientific experiments conducted by the Danish Centre for Remote Sensing (DCRS), and it is expected to be utilized by JRC for its European Airborne Remote Sensing Campaign (EARSEC). Also, EMISAR is expected to be used for the European Multisensor Airborne Campaign (EMAC) arranged by ESA in co-operation with JRC. Some of the data used in this paper were acquired during a calibration experiment sponsored in part by ESA and in part by EMI in order to verify the performance of the SAR-systems which would potentially be used in the EMAC campaigns.

Accurate calibration is essential for most applications of polarimetric SAR data, such as geophysical modelling and parameter retrieval. A number of algorithms has been developed over the last 4-5 years based on distributed natural targets where the fundamental assumption is that the co- and cross-polarized components of the scattering matrix are uncorrelated. This approach was first used by van Zyl (1990), where the radar system was modelled as reciprocal. Klein (1992) removed the restriction about the reciprocal radar system and Yueh et al. (1992) removed the restriction about small cross-talk terms. The above-mentioned algorithms estimate the cross-talk terms using an iterative method, whereas Quegan (1993) derived a direct (i.e. non-iterative) method using the minimum least square principle and the assumption of small cross-talk terms.

Presently, the polarimetric calibration of EMISAR is based on both internal calibration and external calibration, where in the latter case the cross-talk is estimated from distributed targets and the channel imbalance from corner reflectors. The internal polarimetric calibration is presented elsewhere in these proceedings (Dall et al. 1994). In this paper results of applying Klein's and Quegan's algorithms to data from EMISAR will be presented. A new approach to obtain robust estimates from natural targets will also be presented.

0-7803-1497-2/94\$4.00@ 1994 IEEE
Data variations for data acquired on the same mission and on different missions will be analysed. Also, the algorithm results will be compared with results from corner reflectors. The data sets used in this paper are presented in Section 2, and the algorithms are outlined in Section 3. The results are discussed in Section 4, and Section 5 presents our conclusions.

\section{DATA SET}

A total of 3 data sets from two test sites have been used for this study: the DLR calibration area at Oberpfaffenhofen near München, Germany, and the Foulum Research Farm in Jutland, Denmark. The DLR area consists of both agricultural and forest areas, and also a number of large built-up areas. The Foulum area consists of agricultural and forest areas, with only few and small built-up areas. The Foulum area was mapped during a test flight on November 241993 (Foulum- 3 data set), and a number of corner reflectors was deployed at different incidence angles. On December 8, 1993, the DLR test site was mapped (DLR data set) and on the same mission the Foulum area was mapped shortly before landing (Foulum-4 data set).

The data were in all three cases processed to l-look with a spatial resolution of about $2 \mathrm{~m} \times 2 \mathrm{~m}$. The data were stored as scattering matrix data, in slant-range format with 6400 azimuth lines (i.e. all samples in an azimuth line have the same slant range) and 8192 range lines.

\section{CROSS-TALK REMOVAL ALGORITHMS}

Two calibration techniques were evaluated: Klein's iterative method and Quegan's direct method. Both algorithms are designed for scattering matrix data and do not assume the radar system to be reciprocal on transmit and receive. However, they make a number of other assumptions about the distributed targets and the radar system: all scatterers are reciprocal, the like and cross-polarised returns are uncorrelated, the system effects can be modelled by the models shown below, and the cross-talk terms are small. The second assumption is fulfilled for azimuthally symmetric distributed targets, which is for instance rough surfaces (Borgeaud et al., 1987).

The model used by Quegan (1993) to describe the radar system is:

$$
\left[\begin{array}{ll}
o_{h h} & o_{h v} \\
o_{v h} & o_{v v}
\end{array}\right]=\left[\begin{array}{ll}
r_{h h} & r_{h v} \\
r_{v h} & r_{v v}
\end{array}\right]\left[\begin{array}{ll}
s_{h h} & s_{h v} \\
s_{v h} & s_{v v}
\end{array}\right]\left[\begin{array}{ll}
t_{h h} & t_{h v} \\
t_{v h} & t_{v v}
\end{array}\right]
$$

where the matrix accounting for the system noise is excluded. The 0 matrix is the observed scattering matrix measured by the radar system and the $\mathbf{s}$ matrix the ideal scattering matrix for the observed target, where $s_{i j}$ (or $o_{i j}$ ) is the complex scattering amplitude for $i$ receive and $j$-transmit polarisation. The quantity $t_{i j}$ represents the transmission in channel $i$ when channel $j$ is excited, and the quantity $\mathrm{r}_{\mathrm{ij}}$ is the response in channel $\mathrm{i}$ to a stimulus in channel $\mathrm{j}$.

Klein (1992) uses another definition of the elements in the scattering matrix, where $\mathrm{S}_{\mathrm{ij}}\left(\right.$ or $\mathrm{O}_{\mathrm{ij}}$ ) is the complex scattering amplitude for the $\mathrm{i}$-transmit and $\mathrm{j}$-receive polarisation, and the model used is 


$$
\left[\begin{array}{ll}
O_{h h} & O_{h v} \\
O_{v h} & O_{v v}
\end{array}\right]=\left[\begin{array}{ll}
T_{h h} & T_{h v} \\
T_{v h} & T_{v v}
\end{array}\right]\left[\begin{array}{ll}
S_{h h} & S_{h v} \\
S_{v h} & S_{v v}
\end{array}\right]\left[\begin{array}{ll}
R_{h h} & R_{v h} \\
R_{h v} & R_{v v}
\end{array}\right]
$$

where the quantity $T_{i j}$ represents the transmission in channel $j$ when channel $i$ is excited, and the quantity $R_{i j}$ is the response in channel $i$ to a stimulus in channel $\mathrm{j}$, i.e.

$$
t_{i j}=T_{i i}, \quad r_{i j}=R_{i j}
$$

The cross-talk terms estimated by Quegan are

$$
z=\frac{t_{h v}}{t_{h h}}, \quad v=\frac{t_{v h}}{t_{v v}}, \quad w=\frac{r_{h v}}{r_{v v}}, \quad u=\frac{r_{v h}}{r_{h h}}
$$

and by Klein:

$$
\tilde{T}_{h v}=\frac{T_{h v}}{T_{v v}}, \tilde{T}_{v h}=\frac{T_{v h}}{T_{h h}}, \tilde{R}_{h v}=\frac{R_{h v}}{R_{v v}}, \tilde{R}_{v h}=\frac{R_{v h}}{R_{h h}}
$$

The relations between the two sets of terms are then (using (3) - (5))

$$
z=\tilde{T}_{v h}, \quad v=\tilde{T}_{h v}, \quad w=\tilde{R}_{h v}, \quad u=\tilde{R}_{v h}
$$

In order to obtain consistent estimates the cross-talk terms are computed for areas of 8192 complex samples in the azimuth direction and 100 lines in the range direction. After the estimation the cross-talk terms are averaged using moving averaging of 100 terms in range. The estimated cross-talk terms for the DLR data set using the two algorithms are shown in Eigs 1 and 2 .

The estimates of the cross-talk terms are seen to be fluctuating significantly, this is believed to be related to areas, where the assumptions for the algorithms break down. Therefore, a new approach to obtain robust estimates of the cross-talk terms is proposed using the methods based on distributed targets. It is seen from the figures that the cross-talk is rather small, on the order of $-30 \mathrm{~dB}$, therefore the cross-correlation coefficient between the $\mathrm{HH}$ and the HV-channel may be computed without significant error contributions from the cross-talk. The cross-correlation can now be used to exclude the areas where the assumption of uncorrelated likeand cross-polarized returns probably breaks down. A crosscorrelation coefficient mask image is computed using a window size of $5 \times 5$ pixels. A threshold of 0.4 for the correlation coefficient excludes approximately $40 \%$ of the pixels. The resulting estimates for the Quegan and Klein algorithms are shown in Eigs, 3 and 4 respectively. A substantial reduction in the fluctuation of the estimates is observed, and this approach is used for the results shown in the following section.

\section{RESULTS}

To evaluate the data calibrated using Quegan's algorithm using trihedral corner reflectors, we insert the corresponding scattering matrix, which is proportional to the identity matrix in (1)

$$
\left[\begin{array}{ll}
o_{h h} & o_{h v} \\
o_{v h} & o_{v v}
\end{array}\right]=r_{h h} t_{h h}\left[\begin{array}{ccc}
1 & z+w r_{v v} t_{v v} / r_{h h} t_{h h} \\
u+v & r_{v v} t_{v v} / r_{h h} t_{h h} & r_{v v} t_{v v} / r_{h h} t_{h h}
\end{array}\right]
$$

and correspondingly for the Klein algorithm. The maximum value for the off-diagonal elements is obtained when the two terms in the sum have the same phase, and in this case the amplitude will be the sum of the two amplitudes. Therefore, as a first-order approximation the algorithm estimates are compared with the cross-talk terms from the trihedral corner reflectors reduced by $6 \mathrm{~dB}$.

Figs. 5 and 6 show the results from a number of trihedral corner reflectors deployed in the Foulum-3 data set together with the Quegan u-parameter for all three data sets and the Klein $\tilde{\boldsymbol{R}}_{v h}$ parameter for the DLR- and the Foulum- 4 data sets (the algorithm had for unknown reasons convergence problems for the Foulum-3 data set). We observe that the Klein estimates are somewhat larger than the Quegan estimates, and the latter are relatively close to the corner reflector results. Furthermore, we can see from Figs. 3 - 6 that Quegan's algorithm provides more robust estimates of the crosstalk parameters. These results are in agreement with the results found in Skriver et al. (1994) for the AIRSAR data. Consequently, the Quegan algorithm is used for evaluating the cross-talk performance for the EMISAR system.

Fig 7 shows the cross-talk for the Foulum-4 data set. The cross-talk is seen to be virtually independent of range. Also, the cross-talk terms are very stable both between different acquisitions on the same mission (Figs. 3 and 7) and between missions (the u-terms in Fig 5). The cross-talk is low on the order of $-\mathbf{3 0} \mathrm{dB}$ or lower.

In Quegan (1993) the two cross-polarized channels are combined after correction for the cross-talk using the reciprocity principle for the targets, however, in the EMISAR processing system a modified algorithm has been developed to provide both cross-polarized channels after correction. Fig. 8 shows the cross-talk estimates after the correction for the DLR-data set. The cross-talk after correction is below $-38 \mathrm{~dB}$, with a reduction of about $8 \mathrm{~dB}$. The other data sets show similar results, and the results from the trihedral corner reflectors indicate even lower cross-talk

\section{CONCLUSIONS}

The Klein and Quegan algorithms for cross-talk removal using natural distributed targets have been evaluated on three data sets acquired by the EMISAR system. The Quegan algorithm showed the most robust results and the results were similar to corner reflector results. A new approach to obtain robust estimates based on the cross-correlation coefficient between co- and cross-polarized channels has been developed. The cross-talk estimated for the EMISAR data was low (below $-30 \mathrm{~dB}$ ), virtually independent of range, and stable between different acquisitions - within a mission and between missions. The cross-talk was reduced by $8 \mathrm{~dB}$ after correction to less than $-38 \mathrm{~dB}$.

\section{ACKNOWLEDGEMENTS}

This work has been sponsored by the Danish Technical Research Council (STVF) the Danish Space Board and the National Research Council. The data acquisitions were sponsored by the European Space Agency and STVF. Development of the EMISAR system has been co-sponsored by the Thomas B. Thriges Foundation, STVF, the Royal Danish Air Force, the Technical University of Denmark and the Joint Research Centre.

\section{REFERENCES}

Borgeaud, M., R.T. Shin, and J.A. Kong, "Theoretical models for polarimetric radar clutter", J. Electr. Waves and Appl., vol. 1, 1987, pp. 73-89.

Christensen, E.L., S.N. Madsen, J. Dall, N. Skou, K. Woelders, A. Netterstrøm, J.H. Jørgensen. J. Granholm, and M. Dich, "The Danish Polarimetric SAR for Remote Sensing Applications", this issue, 1994

Dall, J., N. Skou, and E.L. Christensen, "Pulse-Based Internal Calibration of Polarimetric SAR", this issue, 1994.

Klein, J., "Calibration of Complex Polarimetric SAR Imagery Using Backscatter Correlations", IEEE Trans. Aerosp. Electr. S yst., vol. 28, 1992, pp. 183-194.

Madsen, S.N., E.L. Christensen, N. Skou, and J. Dall, "The Danish SAR System Design and Initial Tests", IEEE Trans. Geosc. Rem. Sens,. vol. 29, 1991, pp. 417476.

Quegan, S., "A Unified Algorithm for Phase and Cross-talk Calibration of Polarimetric Data - Theory and Observations", IEEE Trans. Geosc. Rem. Sens. subm. to, 1993.

Skriver, H., H.B. Mortensen, and P. Gudmandsen, "Calibration and Modelling of Maestro-1 Polarimetric SAR Data of a Forest Area in Les Landes, France", Int. J. Rem. Sens., accepted for, 1994

Yueh, S.H., S.V. Nghiem, and R Kwok, "External Calibration of Polarimetric Radars Using Distributed Targets", IEEE Trans. Geosc. Rem. Sens,. subm. to. 1992

Zyl, J.J. van, "Calibration of polarimetric radar images using only image parameters and trihedral corner reflector responses", IEEE Trahs. Geosc. Rem. Sens., vol. 28, 1990, pp. 337-348. 


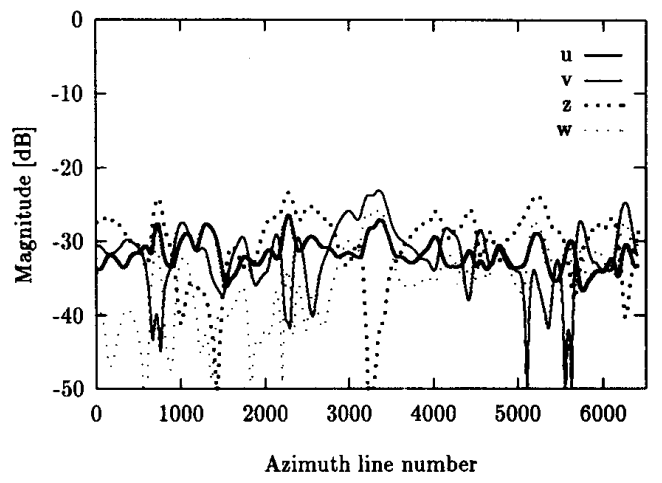

Fig. 1 Quegan's method on DLR data set without mask

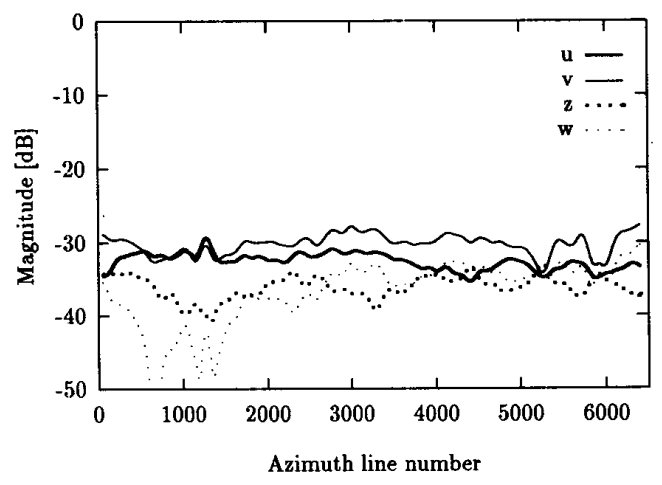

Fig. 3 Quegan's method on DLR data set with mask

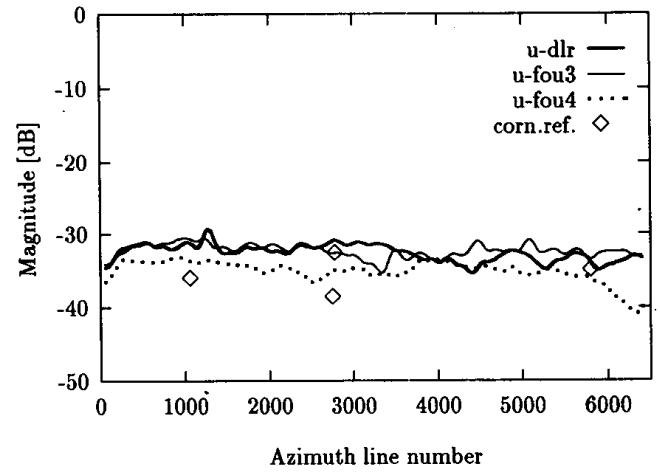

Fig. 5 Comparison of Quegan's u-term with corner reflector results

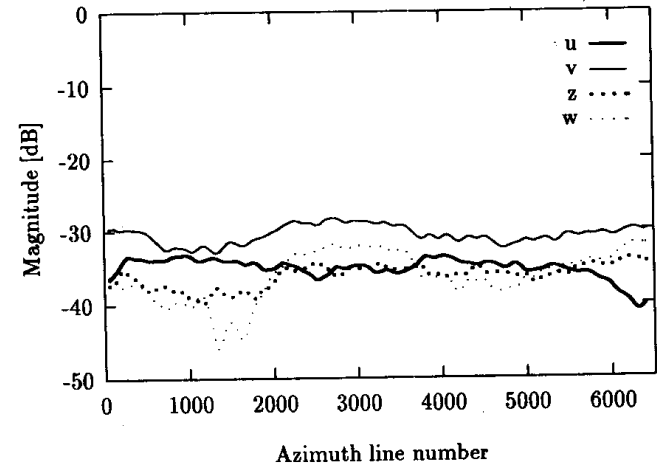

Fig. 7 Quegan's method on Foulum-4 data set

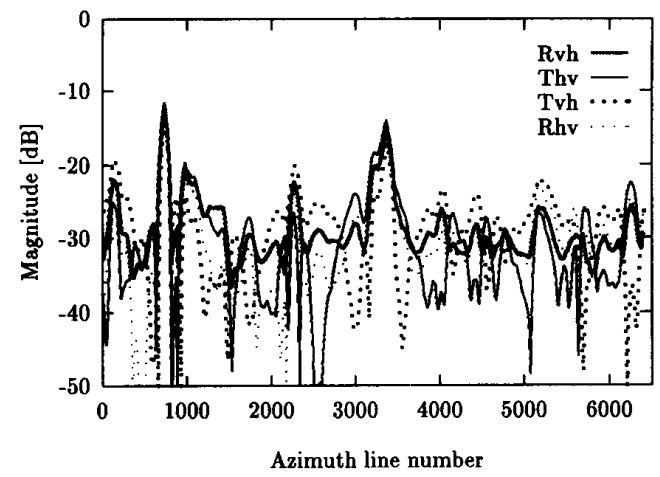

Fig. 2 Klein's method on DLR data set without mask

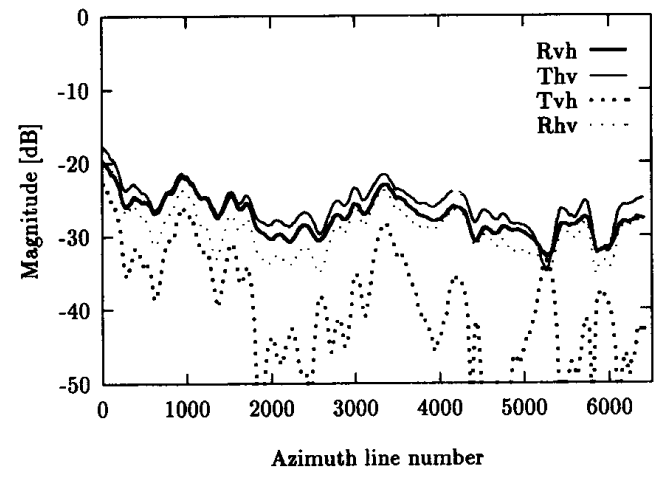

Fig. 4 Klein's method on DLR data set with mask

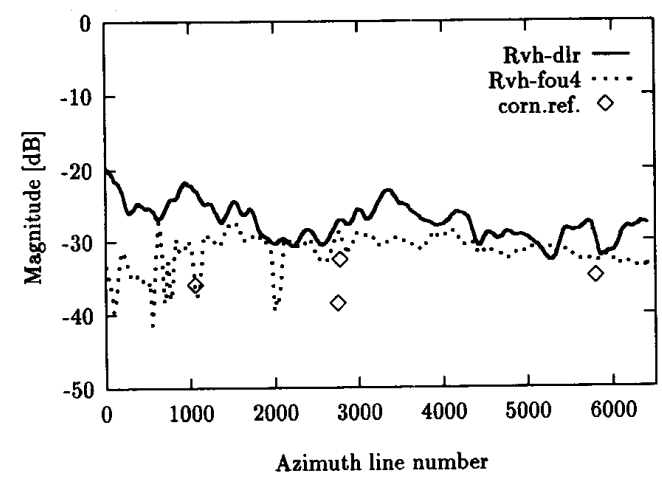

Fig: 6 Comparison of Klein's $\tilde{R}_{v h}$-term with corner reflector results

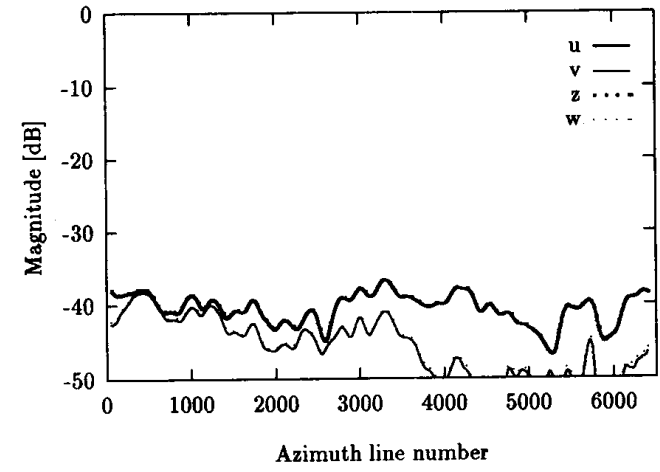

Fig. 8 Cross-talk corrected DLR data set 